\title{
PL Spectra and Hot Charge Carrier Phenomena in an Undoped GaAs/AlGaAs Tunnel-Coupled Quantum Well
}

\author{
Kanothai Jarusirirangsi $\mathbb{D}^{1},{ }^{1}$ Paphavee van Dommelen $\mathbb{D}^{1,2}$ and Chalongrat Daengngam $\mathbb{D}^{1,2}$ \\ ${ }^{1}$ Division of Physical Science, Faculty of Science, Prince of Songkla University, 90110, Hat Yai, Songkhla, Thailand \\ ${ }^{2}$ Thailand Center of Excellence in Physics, Ministry of Higher Education, Science, Research and Innovation, 328 Si Ayutthaya Road, \\ Bangkok 10400, Thailand
}

Correspondence should be addressed to Paphavee van Dommelen; paphavee.t@psu.ac.th

Received 17 June 2021; Accepted 15 July 2021; Published 27 July 2021

Academic Editor: Meiyong Liao

Copyright (c) 2021 Kanothai Jarusirirangsi et al. This is an open access article distributed under the Creative Commons Attribution License, which permits unrestricted use, distribution, and reproduction in any medium, provided the original work is properly cited.

\begin{abstract}
In this research, we experimentally investigated the photoluminescence (PL) spectra of an undoped $\mathrm{GaAs} / \mathrm{Al}_{0.36} \mathrm{Ga}_{0.64} \mathrm{As}^{\mathrm{T}} \mathrm{Tunnel}-$ Coupled Quantum Well (TCQW) at $300 \mathrm{~K}$ and $77 \mathrm{~K}$. At $300 \mathrm{~K}$, PL spectra were broadened at various laser intensities due to the characteristic quantum-confinement electron levels in the active region of the TCQW during pumping excitation. At $77 \mathrm{~K}$, at selected excitation intensities, the high-energy tails in the PL spectra of the studied structure corresponded with hot electron temperatures as functions of the energy of emitted photons. The average scattering energy rate of hot electrons in the system was also studied at a lattice temperature of $77 \mathrm{~K}$. The average scattering energy rate of hot electrons obtained from this experimental study was in agreement with the calculated theoretical value.
\end{abstract}

\section{Introduction}

The lattice constants at the heterojunctions of $\mathrm{GaAs} / \mathrm{Al}_{x} \mathrm{Ga}_{1}$ ${ }_{x}$ As quantum wells are among the most closely matched of all heterostructures. The ease of fabrication of this heterostructure makes it a favored source of radiation for laser and optoelectronics devices emitting in the near-IR range. The IR wavelength region plays important roles in microscopic and macroscopic applications such as molecular spectroscopy combined with Raman spectroscopy [1] and techniques employed in pharmaceutical analysis [2]. However, improving the performance of QW near-IR and mid-IR laser sources is a constant challenge to researchers. The Quantum Cascade Laser (QCL) [3], which is based on intersubband chargecarrier transition, is a familiar source that operates in this spectrum region. However, because the design of QCL is complicated, researchers are always striving to develop simpler QW structures that optimize as much as possible the near-IR and mid-IR output. Among near-IR sources, one of the most applied designs is a Tunnel-Coupled Quantum Well (TCQW) laser of GaAs/ $\mathrm{Al}_{x} \mathrm{Ga}_{1-x}$ As based on the interband transition of carriers. TCQWs have special characteristics of carrier occupation in the active region that encourages population inversion, which is one of the most important features of laser creation.

One approach that has successfully improved the performance of near-IR QW laser sources is the study of hot charge carrier phenomena caused by external perturbation of the lattice. Several experimental and theoretical studies of hot charge carrier phenomena in TCQW structures have reported improved physical properties that enhanced performances. For instance, hot charge carrier phenomena were studied under high laser pumping intensity and electric field application to the lattice [4-9]. These reports of mid-IR and near-IR QW lasers used photoluminescence (PL) spectra to show that hot charge carrier phenomena can improve properties such as interband threshold energy. Moreover, the study of the energy loss rate during hot charge carrier relaxation was an important aspect of investigations into carrier scattering effects on physical properties of nanostructures during external perturbation $[10,11]$. 


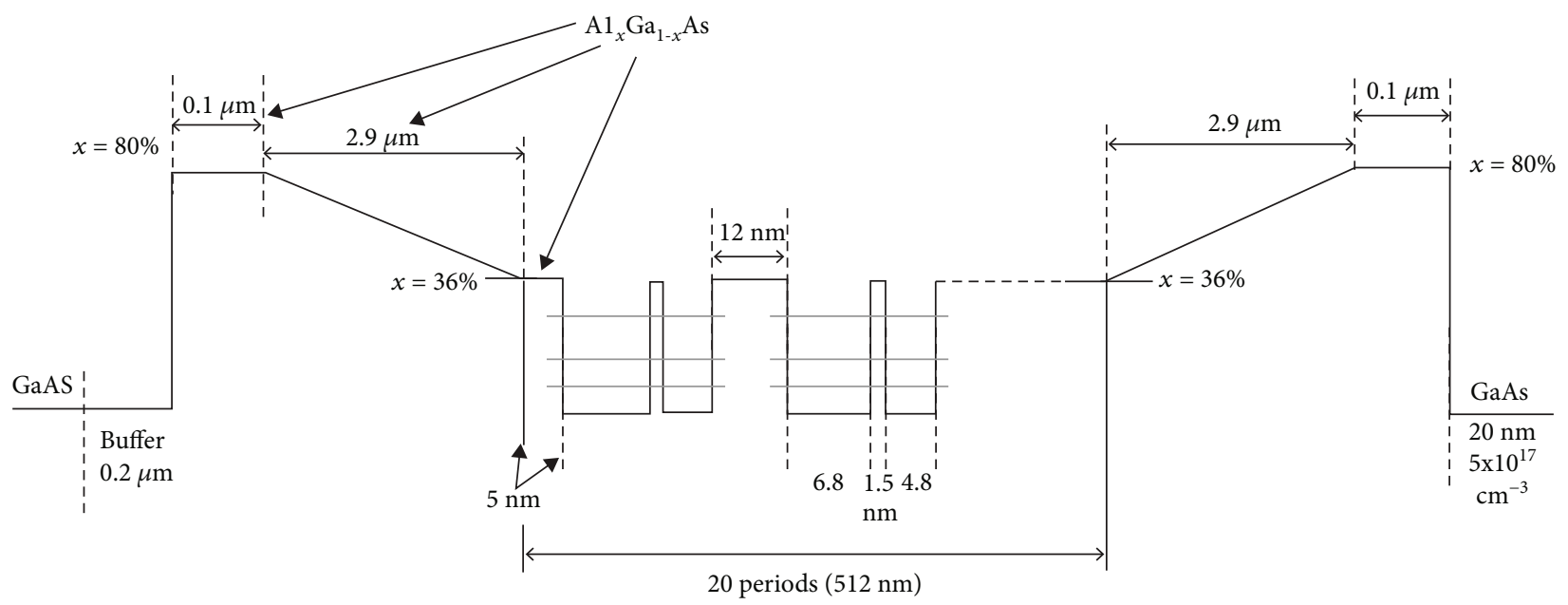

Figure 1: The undoped GaAs/ $\mathrm{Al}_{0.36} \mathrm{Ga}_{0.64}$ As TCQW.

Thus motivated, we propose this experimental research into the PL spectra and hot charge carrier phenomena of an undoped TCQW of GaAs/ $\mathrm{Al}_{x} \mathrm{Ga}_{1-x}$ As. This research is aimed at providing useful information about the influence of external perturbation on the energy levels of carriers in the system, focusing on the scattering energy loss rate of carriers and hot electron temperatures. Therefore, this research can be considered an investigation of the initiation of physical properties of carriers to improve the performance of semiconductor heterostructure IR sources. In addition, research into hot carrier phenomena can introduce innovations which advance nanofabrication technology with the application of electric fields at the Schottky contact of QW structures [12].

\section{Materials and Methods}

The studied structure was an undoped $\mathrm{GaAs} / \mathrm{Al}_{0.36} \mathrm{Ga}_{0.64} \mathrm{As}$ TCQW grown by molecular beam epitaxy (MBE) on a semi-insulating GaAs substrate on a $0.2 \mu \mathrm{m}$ thick GaAs buffer layer (see Figure 1). Its active region consisted of 20 periods of tunnel-coupled GaAs QWs with $\mathrm{Al}_{0.36} \mathrm{Ga}_{0.64} \mathrm{As}$ barriers. The periods comprised GaAs QWs arranged in pairs. One well of each pair was $6.8 \mathrm{~nm}$ wide, and the other was $4.8 \mathrm{~nm}$ wide. The $\mathrm{Al}_{0.36} \mathrm{Ga}_{0.64}$ As layer separating the QWs of each pair was $1.5 \mathrm{~nm}$ wide, and each period was separated by an $\mathrm{Al}_{0.36} \mathrm{Ga}_{0.64} \mathrm{As}$ barrier $12 \mathrm{~nm}$ wide. The capping layer protecting the top $\mathrm{Al}_{0.36} \mathrm{Ga}_{0.64}$ As layer from oxidation was $0.1 \mu \mathrm{m}$ wide.

The fabricated heterostructure was mounted in a variable temperature cryostat (Cryo Industries Variable Temperature Dewar sn 5780), which could vary temperature from $77 \mathrm{~K}$ to $300 \mathrm{~K}$. Two PL experiments were conducted in this research. In the first, PL spectra were produced that displayed PL intensity as a function of laser pumping energy at lattice temperatures of $77 \mathrm{~K}$ and $300 \mathrm{~K}$. In the second experiment, PL was measured under a pumping intensity of $23.8 \mathrm{~kW} / \mathrm{m}^{2}$ at various lattice temperatures from $77 \mathrm{~K}$ to $300 \mathrm{~K}$. The studied structure was excited by a continuous-wave (CW) laser producing a wavelength of $532 \mathrm{~nm}$. The temperature range was controlled between $77 \mathrm{~K}$ and $300 \mathrm{~K}$ by a Lakeshore Model 335 temperature controller. The near-IR interband PL signals were collected and focused into a spectrometer. The experimental setup is shown in Figure 2.

\section{Results and Discussion}

3.1. PL Spectrum Measurement at $300 \mathrm{~K}$ and $77 \mathrm{~K}$ with Varied Laser Pumping Intensities. At $300 \mathrm{~K}$, the PL spectrum of the studied structure presented three emission peaks (see Figure 3). The quantum-confinement energy levels were calculated based on the quantum well widths of the TCQW structure, which were $6.8 \mathrm{~nm}$ and $4.8 \mathrm{~nm}$. A similar structure was described by Firsov et al. [6]. The peak at $1.465 \mathrm{eV}$ conformed to the interband transition of electrons from electron subband level $\mathrm{e}_{1}$ to holes at heavy hole subband level $\mathrm{hh}_{1}$ of the TCQW region $\left(\mathrm{e}_{1}-\mathrm{hh}_{1}\right)$. The peak at $1.508 \mathrm{eV}$ corresponded to transition from the $\mathrm{e}_{2}$ level to the $\mathrm{hh}_{2}$ level $\left(\mathrm{e}_{2}\right.$ $\mathrm{hh}_{2}$ ). The remaining peak at $1.478 \mathrm{eV}$ (peak number 2) was attributed to the transition of carriers from electron subband level $e_{1}$ to holes at light hole subband level $\operatorname{lh}_{1}\left(e_{1}-1 h_{1}\right)$. Our assumption was that broadening of the spectrum took place in the high-energy regions of emission because of the increased electron concentrations at level $e_{2}$. Therefore, the $\mathrm{PL}$ spectrum at $300 \mathrm{~K}$ presented three transitions in superposition. To confirm this assumption, the Lorentzian contours were applied (inset Figure 3). The three peaks in the PL spectrum in Figure 3 corresponded well to the three transitions $\left(e_{1}-h h_{1}, e_{1}-l h_{1}\right.$, and $\left.e_{2}-h h_{2}\right)$ and fitted well with the decomposed curves produced by Lorentzian approximation. The quantum-confinement energy levels and transitions in the active region of the TCQW at $300 \mathrm{~K}$ were presented schematically in an energy band diagram (see Figure 4).

To investigate hot charge carrier phenomena of the studied structure, the temperature of the lattice structure was reduced to a constant $77 \mathrm{~K}$ because it is difficult to observe hot electron temperature at the high-energy tail of PL spectra produced at a lattice temperature of $300 \mathrm{~K}$. PL spectra were then produced at various optical pumping intensities. Under a pumping intensity of $81.07 \mathrm{~kW} / \mathrm{m}^{2}$, PL peaks occurred at 


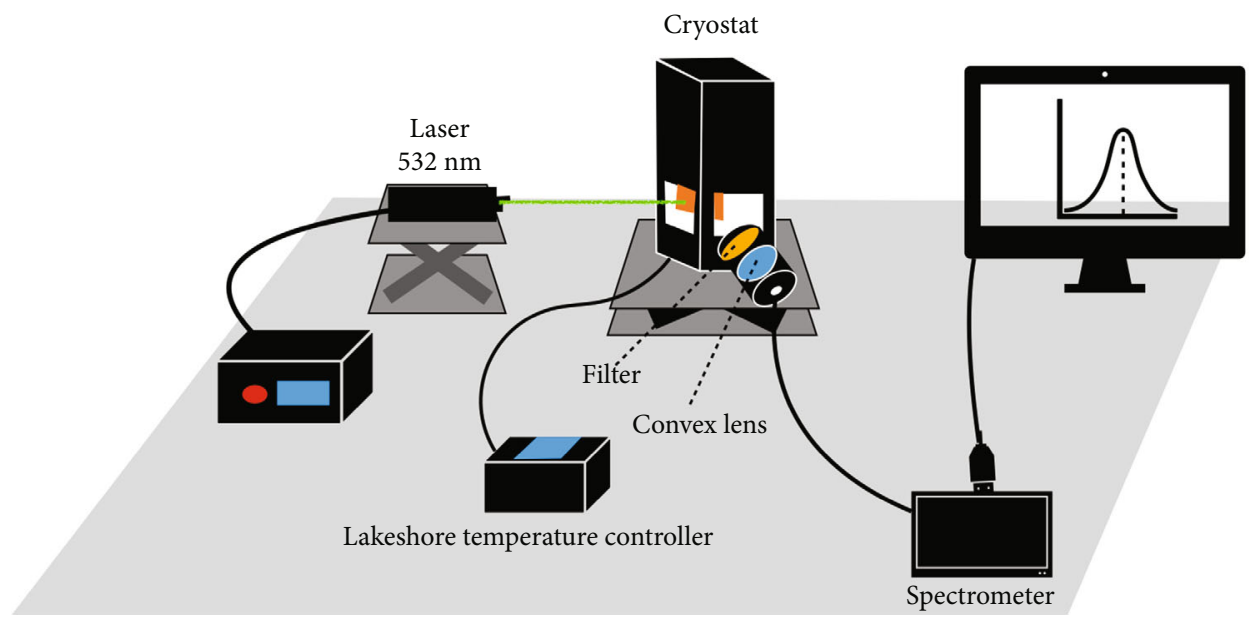

Figure 2: PL experimental setup.

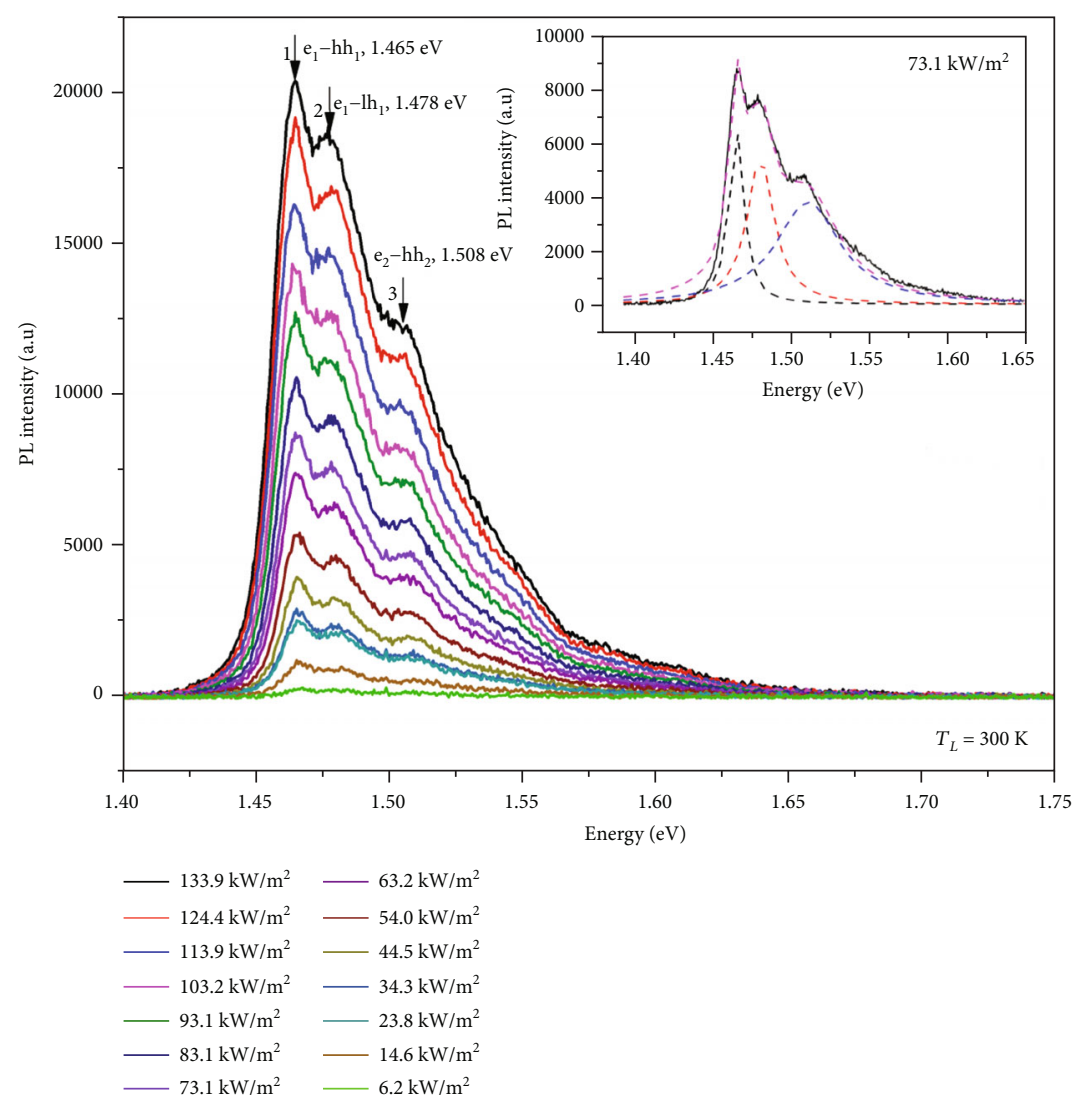

FIGURE 3: The PL spectra were produced by an undoped $\mathrm{GaAs} / \mathrm{Al}_{0.36} \mathrm{Ga}_{0.64} \mathrm{As}$ TCQW at $300 \mathrm{~K}$ under various laser pumping intensities. The arrows above peaks 1,2 , and 3 of the signals indicate the interband transition of $\mathrm{e}_{1}-\mathrm{hh}_{1}, \mathrm{e}_{1}-\mathrm{lh}_{1}$, and $\mathrm{e}_{2}-\mathrm{hh}_{2}$, respectively. The inset shows the Lorentzian contours as three dash lines.

$1.555 \mathrm{eV}$ and $1.568 \mathrm{eV}$ (see Figure 5). Based on the calculated quantum-confinement energy levels and squares of envelope wave functions (see Figure 4), we concluded that these PL peaks corresponded to the $\mathrm{e}_{1}-\mathrm{hh}_{1}$ and $\mathrm{e}_{2}-\mathrm{hh}_{2}$ transitions, respectively.

Under the influence of a sufficiently intense optical excitation, the lattice temperature of a TCQW will rise, and free carriers will have more kinetic energy. To maintain the stationary energy state of the system, these free carriers transfer their excess energy back to the system through collisions with other carriers or by emitting and absorbing optical phonons. These events are known as hot charge carrier phenomena. Since we had obtained information about hot electron temperatures from the high-energy tails of PL spectra produced 


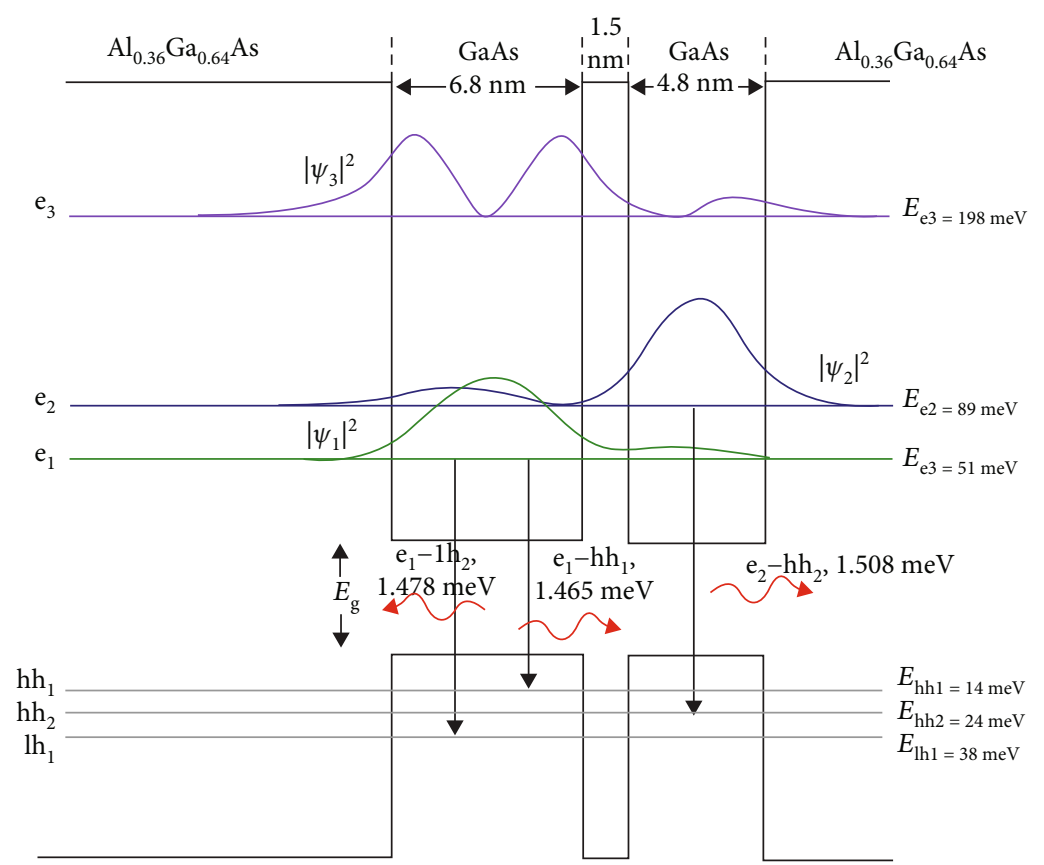

FIGURE 4: Schematic of carrier transitions in the active region of the undoped $\mathrm{GaAs} / \mathrm{Al}_{0.36} \mathrm{Ga}_{0.64} \mathrm{As}$ TCQW under interband optical pumping at a lattice temperature of $300 \mathrm{~K}$. The calculated quantum-confinement energy levels and the squares of envelope wave functions are also presented.

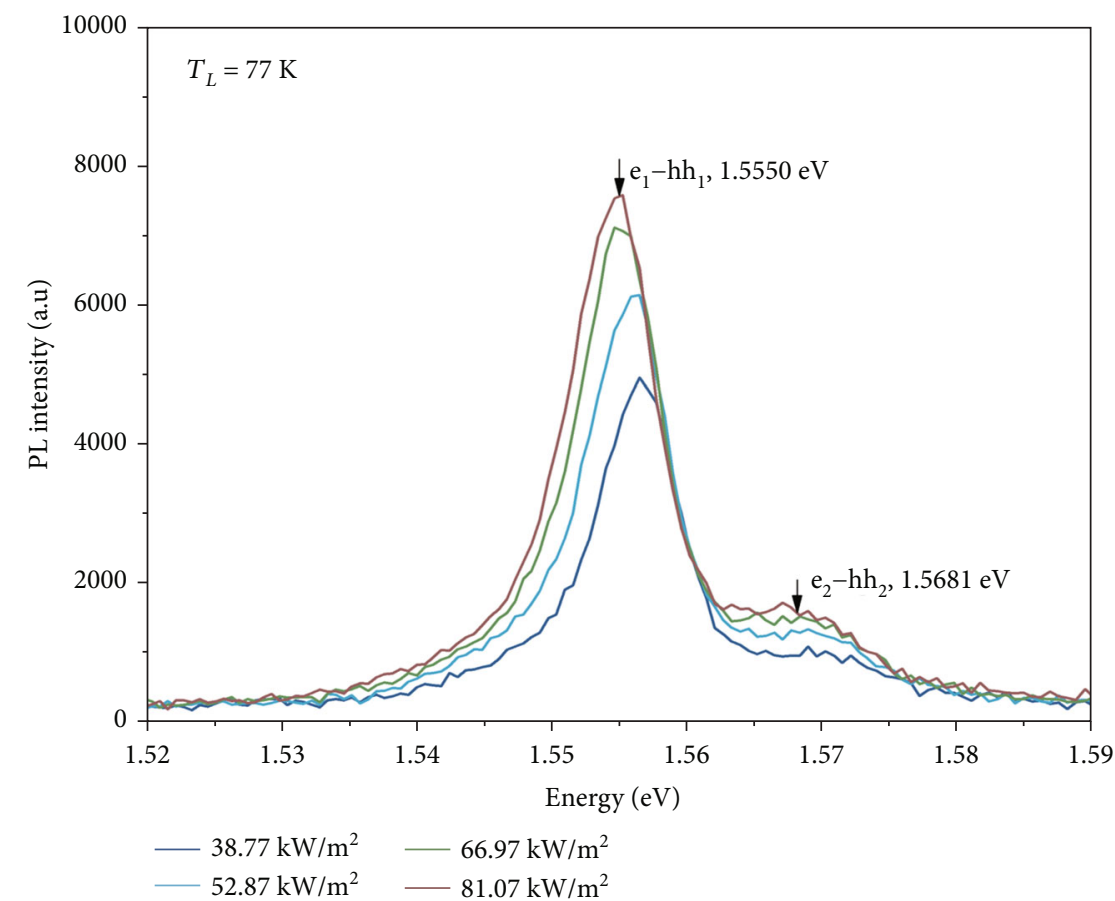

FIGURE 5: The PL spectra of an undoped $\mathrm{GaAs} / \mathrm{Al}_{0.36} \mathrm{Ga}_{0.64} \mathrm{As}$ TCQW were recorded at a lattice temperature of $77 \mathrm{~K}$ using various laser pumping intensities. The arrows above signal peaks indicate energy levels of interband transitions between electrons and heavy holes $\mathrm{e}_{1}-\mathrm{hh}_{1}$ and $\mathrm{e}_{2}-\mathrm{hh}_{2}$.

at a lattice temperature of $77 \mathrm{~K}$, we recorded PL spectra produced at the same lattice temperature under selected laser pumping intensities and then considered the high-energy tails as functions of the energy of photons emitted at $77 \mathrm{~K}$ (see Figure 6).
The hot electron temperatures $\left(T_{e}\right)$ derived from the high-energy tails of the obtained PL spectra were higher than the lattice temperature $\left(T_{L}\right)$. To calculate hot electron temperature, the classical Maxwel-Boltzmann distribution must be considered. Then, $T_{e}$ can be defined as a function of PL 


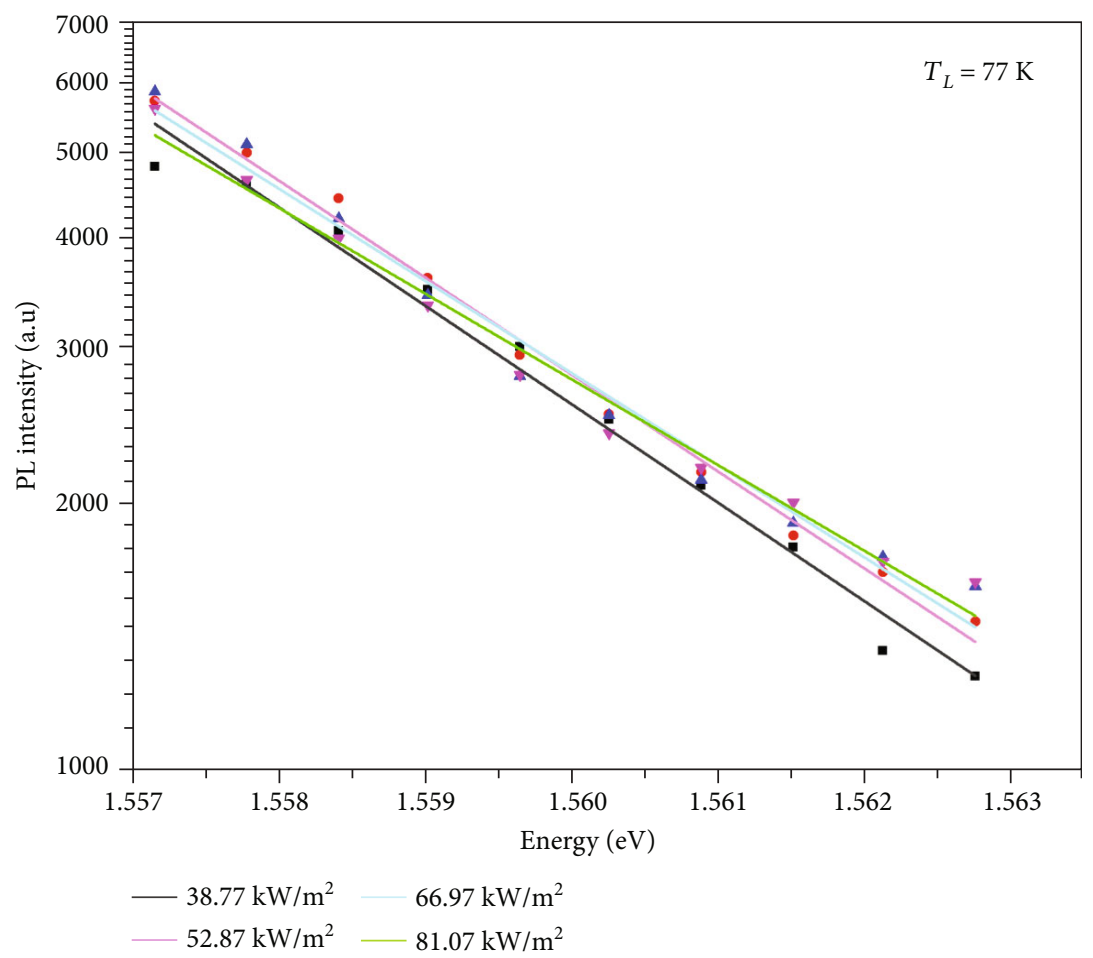

FIGURE 6: The high-energy tails of PL spectra at $77 \mathrm{~K}$ at selected laser pumping intensities were plotted in semilogarithm scale.

intensity $\left(I_{\mathrm{PL}}\right)$ from Equation (1) [13].

$$
I_{\mathrm{PL}}=I_{0} e^{\left(-h v / k_{B} T_{e}\right)},
$$

where $I_{0}$ is the laser pumping intensity absorbed by the sample at the maximum excitation intensity, $h v$ is the photon energy emitted from the studied structure, and $k_{B}$ is the Boltzmann constant. Based on Equation (1), the experimental data obtained at pumping intensities from 38.77 to $81.07 \mathrm{~kW} / \mathrm{m}^{2}$ yielded hot electron temperatures ranging from 105 to $120 \mathrm{~K}$.

The dependence of the average scattering energy rate on the temperature of hot electrons was then applied to calculate how much energy one electron transferred to the lattice via scattering processes during a given interval of time. This specific dependence was calculated from the power balance equation [14].

$$
\left\langle\frac{d \varepsilon}{d t}\right\rangle=I_{\text {pump }} \cdot \frac{h v-E_{g}^{*}}{h v} \cdot \frac{1}{n^{*}},
$$

where $I_{\text {pump }}$ was the laser pumping intensity, $h v$ was the photon energy of the laser output at $532 \mathrm{~nm}, E_{g}^{*}$ was the energy band gap calculated from interband transition $\mathrm{e}_{1}-\mathrm{hh}_{1}$, and $n^{*}$ was the concentration of surface electrons in the excited electron system after the loss of excess energy through electron-electron interaction and polar longitudinal optical phonon emission.

However, if the energy of photoexcited electrons was much higher than polar longitudinal optical phonon energy, $n^{*}$ could be defined as

$$
n^{*}=|e| E_{0} \cdot \hbar \omega_{L O} \frac{\left(\varepsilon_{\infty}\right)^{2}}{4 \pi e^{4}} \cdot b \cdot \ln \left[4 \cdot \frac{h v-E_{g}^{*}}{\hbar \omega_{L O}}\right]
$$

where $\hbar \omega_{L O}$ is the polar longitudinal optical phonon energy ( $36 \mathrm{meV}$ in $\mathrm{GaAs}$ ) and $b$ is the width of the wider QW of the TCQW $(6.8 \mathrm{~nm}$ in this structure). Note that, in the absence of light, the total density of conduction-subband electrons in the TCQW from the initial subband $\left(e_{i}\right)$ to the final subband $\left(e_{f}\right)$ could be calculated as

$$
n_{0}=\int_{e_{i}}^{e_{f}} g(E) f(E)
$$

where $g(E)=m / \pi \hbar^{2}$ is the density of states of a twodimensional electron gas and $f(E)$ is the Fermi-Dirac distribution function. Further, in the beginning of the optical excitation process, before reaching the threshold excitation for laser generation, $n_{0}$ is proportional to the intensity of pumping radiation,

$$
n_{0} \alpha I_{\text {pump }}
$$

Therefore, since the electron concentrations in $\mathrm{e}_{1}$ and $\mathrm{e}_{2}$ are proportional to $I_{\text {pump }}$, charge carriers need to release their excess energy by scattering, either in collisions with other carriers or through releasing optical phonons into the system. From the power balance Equations (2) and (3) above, the relaxing (or scattering) process of carriers takes place on a picosecond timescale. 


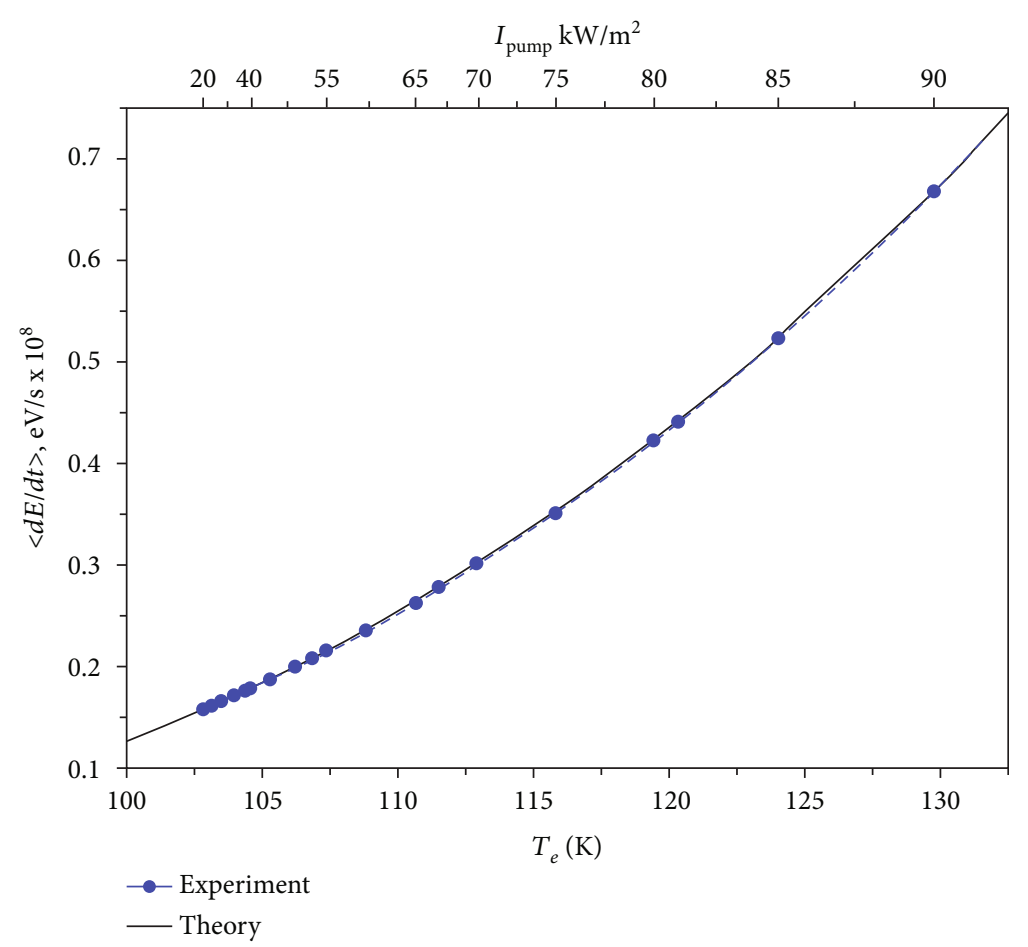

Figure 7: The plot shows $\langle d \varepsilon / d t\rangle\left(T_{e}\right)$ obtained experimentally and $\langle d \varepsilon / d t\rangle\left(T_{e}\right)$ obtained theoretically as a function of hot electron temperatures. The values are presented as a circle-plot and a bold line, respectively. The laser pumping intensity is also shown.

The average scattering energy rate as a function of the hot electron temperatures for our structure at a $T_{L}$ of $77 \mathrm{~K}$ could now be theoretically determined as follows [15]:

$$
\begin{aligned}
& \left\langle\frac{d \varepsilon}{d t}\right\rangle\left(T_{e}\right)=\sqrt{\frac{2 \hbar \omega_{L O}}{\pi m^{*}}} \cdot|e| E_{0} \\
& \cdot \frac{\exp \left(-x_{C}\right)-\exp \left(-x_{L}\right) \sqrt{\left(0.5 x_{C}\right)} \exp \left(0.5 x_{C}\right) K_{0}\left(0.5 x_{C}\right)}{\left(1-\exp \left(-x_{L}\right)\right) \sqrt{\pi / 2}}
\end{aligned}
$$

where $|e| E_{0}=m^{*} e^{2} \hbar \omega_{L O} / \hbar^{2} \cdot\left(1 / \varepsilon_{\infty}-1 / \varepsilon_{0}\right) ; e$ is the electron charge; $m^{*}=0.063 m_{0}$, the effective electron mass for GaAs; $m_{0}=9.1 \times 10^{-31} \mathrm{~kg} ; \hbar$ is Planck's constant; $\varepsilon_{\infty}$ and $\varepsilon_{0}$ are, respectively, the high-frequency and static dielectric constants; $x_{C}=\hbar \omega_{\mathrm{LO}} / k_{B} T_{e} ; x_{L}=\hbar \omega_{L O} / k_{B} T_{L} ; T_{L}$ is the lattice temperature $=77 \mathrm{~K} ; T_{e}$ is the hot electron temperature, and $K_{0}\left(0.5 x_{C}\right)$ is the modified Bessel function of the order zero. The $\hbar \omega_{\mathrm{LO}}$ energy is presented here, because, in this studied structure, the difference in quantum-confinement energy levels between the first electron level, $\mathrm{e}_{1}$, and the second electron level, $\mathrm{e}_{2}$, is about $38 \mathrm{meV}$. This energy difference is about the same as the $\hbar \omega_{\mathrm{LO}}$ energy. As a result, when excited, electrons located at $e_{1}$ can transfer to $e_{2}$ of the narrower QW of the TCQW by the process of optical phonon absorption.

As discussed above, using Equation (1) and data from the high-energy tails of the experimentally obtained PL spectra (see Figure 6), we obtained $T_{e}$ ranging from 105 to $120 \mathrm{~K}$. Using Equation (6), $\langle d \varepsilon / d t\rangle\left(T_{e}\right)$ was theoretically calculated from the derived data as a function of hot electron tempera- tures. The $\langle d \varepsilon / d t\rangle\left(T_{e}\right)$ obtained from experiment varies exponentially, thus in agreement with theoretical expectation. The results of the calculations from experimental and theoretical data were presented in Figure 7.

3.2. PL Spectrum Measurement at a Constant Pumping Intensity at Various Lattice Temperatures from $77 \mathrm{~K}$ to $300 \mathrm{~K}$. To investigate the hot charge carrier phenomena of the undoped $\mathrm{GaAs} / \mathrm{Al}_{0.36} \mathrm{Ga}_{0.64} \mathrm{As}$ TCQW at different lattice temperatures, PL intensity was measured at a constant pumping intensity of $23.8 \mathrm{~kW} / \mathrm{m}^{2}$ while lattice temperature was varied from $77 \mathrm{~K}$ to $300 \mathrm{~K}$ by increments of approximately $10 \mathrm{~K}$. The experimental results showed that at lattice temperatures higher than $96.7 \mathrm{~K}$ (see Figure 8), the interband transition peaks of $\mathrm{e}_{1}-\mathrm{hh}$, were shifted to the low-energy region because of heating in the active region of the structure during pumping.

The redshift behavior can be explained by the behavior of the energy band gap $\left(E_{g}\right)$ of GaAs. The energy band gap of $\mathrm{GaAs}$ as a function of lattice temperature was defined by the following equation:

$$
E_{g}\left(T_{L}\right)=1.519-\frac{5.405 \times 10^{-4} T_{L}^{2}}{T_{L}+204},
$$

where $T_{L}$ was varied from $77 \mathrm{~K}$ to $300 \mathrm{~K}$. It is clear from Equation (7) [16] that $E_{g}$ decreases when $T_{L}$ increases, and consequently, PL peak intensity decreases with increments of lattice temperature. Therefore, at a lattice temperature of $300 \mathrm{~K}$, the $E_{g}\left(T_{L}\right)$ of GaAs was narrower than it was at $77 \mathrm{~K}$. 


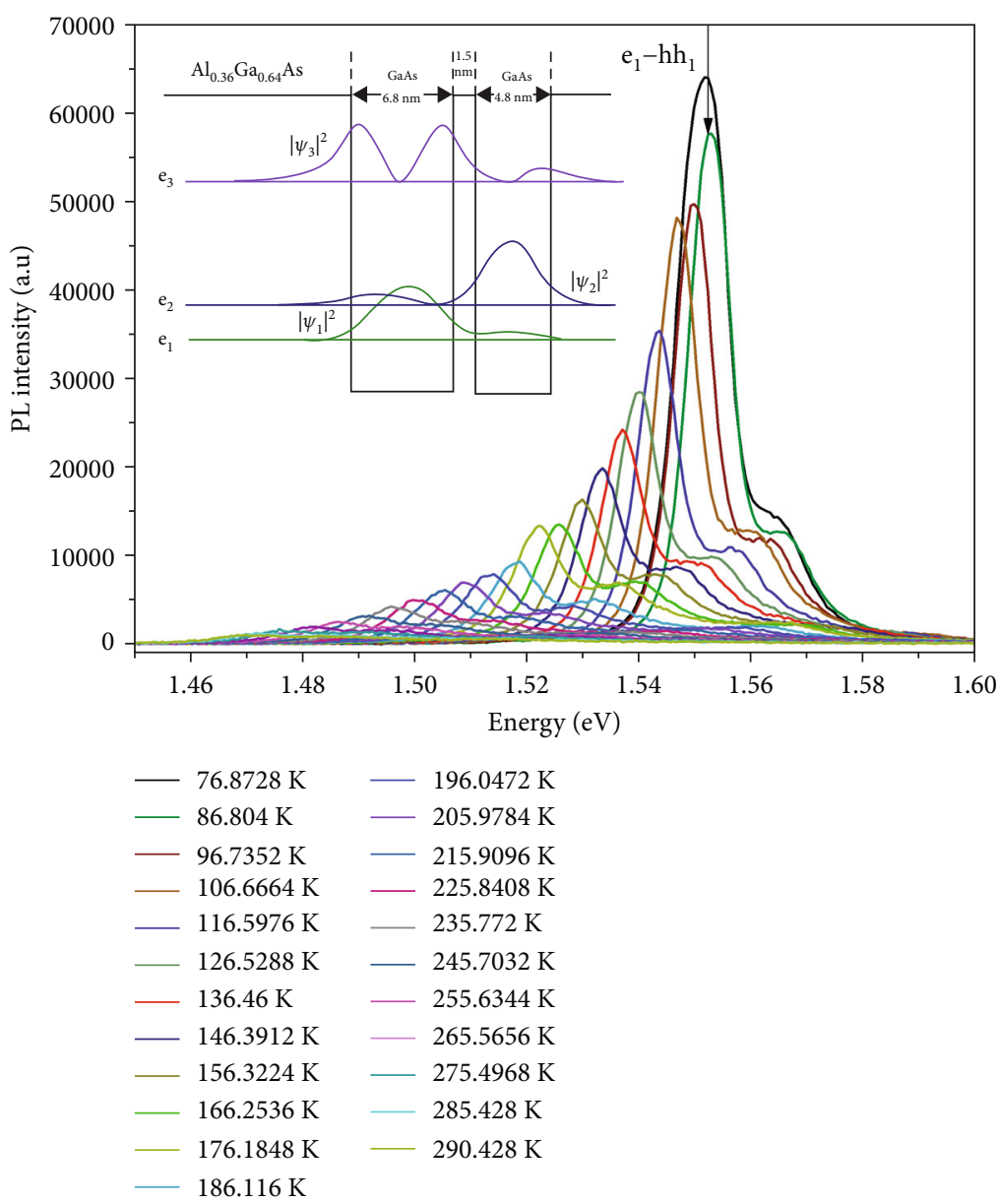

Figure 8: The PL spectra of the undoped $\mathrm{GaAs} / \mathrm{Al}_{0.36} \mathrm{Ga}_{0.64} \mathrm{As} \mathrm{QW}$ were produced at a pumping intensity of $23.8 \mathrm{~kW} / \mathrm{m}^{2}$ under lattice temperature variation from $77 \mathrm{~K}$ to $300 \mathrm{~K}$. The square of the envelope functions of the first $\left(\left|\psi_{1}\right|^{2}\right)$, second $\left(\left|\psi_{2}\right|^{2}\right)$ and third $\left(\left|\psi_{3}\right|^{2}\right)$ electron subbands are also presented.

Besides the redshift behavior of $\mathrm{e}_{1}-\mathrm{hh}_{1}$ transition, the interband transition peak intensity of $\mathrm{e}_{1}-\mathrm{h} \mathrm{h}_{1}$ was higher at $77 \mathrm{~K}$ than at $300 \mathrm{~K}$ at the same laser pumping intensity. The main reason for this higher transition energy was the difference in the quantum-confinement energy levels of $e_{1}$ and $e_{2}$, which was about $38 \mathrm{meV}$. This energy difference is not much more than thermal energy at room temperature $(26 \mathrm{meV})$. Also, the square of the envelope wave functions of the first $\left(\left|\psi_{1}\right|^{2}\right)$ and second $\left(\left|\psi_{2}\right|^{2}\right)$ subbands of the first electron level $\mathrm{e}_{1}$ and the second electron level $\mathrm{e}_{2}$ were, respectively, located at the wider QW and at the narrower QW of the TCQW structure. Therefore, at room temperature under sufficiently high interband optical pumping, electrons can possibly occupy levels $e_{1}$ and $e_{2}$ at the same time; consequently, the $\mathrm{e}_{1}-\mathrm{hh}_{1}$ peak intensity was lower at $300 \mathrm{~K}$ than at $77 \mathrm{~K}$. The squares of the envelope functions of $\mathrm{e}_{1}\left(\left|\psi_{1}\right|^{2}\right)$, $\mathrm{e}_{2}\left(\left|\psi_{2}\right|^{2}\right)$, and $\mathrm{e}_{3}\left(\left|\psi_{3}\right|^{2}\right)$ are presented in the inset of Figure 8 .

\section{Conclusions}

We investigated the intensity of photoluminescence spectra produced by an undoped $\mathrm{GaAs} / \mathrm{Al}_{0.36} \mathrm{Ga}_{0.64} \mathrm{As}$ Tunnel-
Coupled Quantum Well under various laser pumping intensities at lattice temperatures of $77 \mathrm{~K}$ and $300 \mathrm{~K}$. At a lattice temperature of $300 \mathrm{~K}$, all photoluminescence spectra showed three peaks corresponding to interband transitions, which were electron-hole transitions from $e_{1}-h_{1}$, from $e_{2}-h h_{2}$, and from $e_{1}-h_{1}$. The three transitions fitted well with Lorentzian curves. Broadening of the spectrum was discussed as a possible indication of injected electrons at subband level $e_{2}$. The proposition was confirmed by the calculated quantumconfinement energy and the square of the envelope wave functions of electron subband levels in the active region of the TCQW. At a lattice temperature of $77 \mathrm{~K}$, photoluminescence spectra showed two peaks that corresponded to $\mathrm{e}_{1}$ $\mathrm{hh}_{1}$ and $\mathrm{e}_{2}-\mathrm{hh}_{2}$ electron-hole transitions. These transition peaks shifted to the low-energy region as laser pumping intensity increased. The slight shift in the peak $\mathrm{e}_{1}-\mathrm{hh} \mathrm{h}_{1}$ from a higher energy region to a lower energy region of emission was a result of heat transfer due to air convection in the cryostat during the continuous laser pumping excitation of the sample. High laser pumping intensity affected the physical properties of carriers in the system, increasing their temperature, thus creating hot electrons. Hot electron temperature in this structure was investigated by studying high-energy tail 
photoluminescence intensity. The results of the average scattering energy rate at a lattice temperature of $77 \mathrm{~K}$ as a function of hot electron temperatures were compared with results obtained from theoretical calculations. Finally, we experimentally studied the photoluminescence intensity spectra produced at lattice temperatures from $77 \mathrm{~K}$ to $300 \mathrm{~K}$ under a fixed pumping intensity of $23.8 \mathrm{~kW} / \mathrm{m}^{2}$. At higher lattice temperatures, characteristic photoluminescence peak broadening occurred, producing interband transition peaks from $\mathrm{e}_{1}-\mathrm{hh}$ that had higher intensity at $77 \mathrm{~K}$ than at $300 \mathrm{~K}$.

\section{Data Availability}

The calculation of quantum-confinement energy level data used to support the findings of this study are included within the article.

\section{Conflicts of Interest}

The authors declare that there are no conflicts of interest.

\section{Acknowledgments}

This research was supported by the Program Management Unit for Human Resources \& Institutional Development, Research and Innovation (grant number B05F630108), the Thailand Excellence Center in Physics (ThEPCenter) (grant number ThEP-60-PHY-PSU4), and the Development and Promotion of Science and Technology Talents Project (DPST). The fabrication of the TCQW and the calculation of quantum-confinement energy levels were carried out at the Nonequilibrium Electrons Optics Laboratory, Institute of Physics, Nanoelectronics and Telecommunications, Peter the Great Saint Petersburg Polytechnic University, Russian Federation. The authors would like to thank Dr. Komrit Wattanavatee for supplying the $\mathrm{LN}_{2}$ used in this research. Mr. Thomas Duncan Coyne helped with the English grammatical revision.

\section{References}

[1] M. A. Czarnecki, Y. Morisawa, Y. Futami, and Y. Ozaki, "Advances in molecular structure and interaction studies using near-infrared spectroscopy," Chemical Reviews, vol. 115, no. 18, pp. 9707-9744, 2015.

[2] Y. Roggo, P. Chalus, L. Maurer, C. Lema-Martinez, A. Edmond, and N. Jent, "A review of near infrared spectroscopy and chemometrics in pharmaceutical technologies," Journal of Pharmaceutical and Biomedical Analysics, vol. 44, no. 3, pp. 683-700, 2007.

[3] J. Faist, F. Capasso, D. L. Sivco, C. Sirtori, A. L. Hutchinson, and A. Y. Cho, "Quantum cascade laser," Science, vol. 264, no. 5158, pp. 553-556, 1994.

[4] V. L. Zerova, G. G. Zegrya, and L. E. Vorob'ev, "Effect of electron-electron and electron-hole collisions on intraband population inversion of electrons in stepped quantum wells," Semiconductors, vol. 38, no. 9, article 1936, pp. 1053-1060, 2004.

[5] L. E. Vorobjev, M. Y. Vinnichenko, D. A. Firsov et al., "Carrier heating in quantum wells under optical and current injection of electron-hole pairs," Semiconductors, vol. 44, no. 11, pp. 1402-1405, 2010.

[6] D. A. Firsov, L. E. Vorobjev, M. Y. Vinnichenko, R. M. Balagula, M. M. Kulagina, and A. P. Vasil'iev, "Effect of transverse electric field and temperature on light absorption in GaAs/AlGaAs tunnel-coupled quantum wells," Semiconductors, vol. 49, no. 11, pp. 1425-1429, 2015.

[7] L. E. Vorob'ev, S. N. Danilov, V. L. Zerova, and D. A. Firsov, "Electron heating by a strong longitudinal electric field in quantum wells," Semiconductors, vol. 37 , no. 5, pp. 586-593, 2003.

[8] L. E. Vorob'ev, V. L. Zerova, K. S. Borshchev, Z. N. Sokolova, I. S. Tarasov, and G. Belenky, "Charge-carrier concentration and temperature in quantum wells of laser heterostructures under spontaneous and stimulated-emission conditions," Semiconductors, vol. 42, no. 6, article 6017, pp. 737-745, 2008.

[9] P. van Dommelen, "Drift velocity and the rate of carrier scattering energy in Sb-containing heterostructure lasers," Superlattices and Microstructures, vol. 98, pp. 140-147, 2016.

[10] B. Roy, S. Bhattacharyya, and D. P. Bhattacharya, "Heating of a degenerate electron ensemble in a well of compound semiconductors at low lattice temperatures," Applied Physics A: Materials Science \& Processing, vol. 125, no. 3, article 2494, pp. 223 235, 2019.

[11] B. Roy, S. Bhattacharyya, and D. P. Bhattacharya, "Energy loss rate and non-ohmic characteristics of a degenerate surface layer of compound semiconductors at low lattice temperatures," Physica E: Low-dimensional Systems and Nanostructures, vol. 126, article 114465, 2021.

[12] A. Furube and S. Hashimoto, "Insight into plasmonic hotelectron transfer and plasmon molecular drive: new dimensions in energy conversion and nanofabrication," NPG Asia Materials, vol. 9, no. 12, article e454, 2017.

[13] J. Shah and R. C. C. Leite, "Radiative recombination from photoexcited hot carriers in GaAs," Physical Review Letters, vol. 22, no. 24, pp. 1304-1307, 1969.

[14] L. E. Vorobjev, S. N. Danilov, E. L. Ivchenko, M. E. Levinstein, D. A. Firsov, and V. A. Shalygin, Kinetics and optical phenomena in strong electric field in semiconductors and nanostructures, Nauka, St. Petersburg, 2000.

[15] J. Shah, A. Pinczuk, A. C. Gossard, and W. Wiegmann, "Energy-loss rates for hot electrons and holes in GaAs quantum wells," Physical Review Letters, vol. 54, no. 18, pp. 20452048, 1985.

[16] http://www.ioffe.ru/SVA/NSM/Semicond/GaAs/bandstr .html\#Temperature/. 